

\title{
Efficacy of Off-Label Topical Treatments for the Management of Androgenetic Alopecia: a Systematic Review
}

Aditya K. Gupta M.D., Ph.D. ${ }^{1,2}$, Rachel R. Mays B.Sc. ${ }^{1}$, Sarah G Versteeg M.Sc. ${ }^{1}$, Neil H. Shear M.D. ${ }^{2,3}$, Vincent Piguet M.D., Ph.D. ${ }^{2,4,5}$ and Bianca Maria Piraccini M.D. ${ }^{6}$

\author{
${ }^{1}$ Mediprobe Research Inc., London, Canada \\ ${ }^{2}$ Division of Dermatology, Department of Medicine, University of Toronto School of Medicine, Toronto, Canada \\ ${ }^{3}$ Division of Dermatology, Sunnybrook Health Sciences Centre, Toronto, Canada \\ ${ }^{4}$ Division of Dermatology, Women's College Hospital, Toronto, Canada \\ ${ }^{5}$ Division of Infection and Immunity, Cardiff University School of Medicine, Cardiff, United Kingdom \\ ${ }^{6}$ Dermatology, Department of Experimental, Diagnostic and Specialty Medicine, University of Bologna, Italy
}

\section{Corresponding Author:}

A.K. Gupta

Mediprobe Research Inc.

645 Windermere Road

London, Ontario, Canada N5X 2P1

Phone: 519-851-9715

Fax: 519-657-4233

Email: agupta@mediproberesearch.com

\section{Compliance with Ethical Standards}

Funding: This paper was not funded.

Conflict of interest: Dr. Piguet reports receiving educational grants in his role as Department Division Director, Dermatology, University of Toronto (on behalf of the Division of Dermatology Residency Program) from Abbvie, Celgene, Janssen, Naos, Lilly, Sanofi, Valeant, and non-financial support from La Roche-Posay, outside the submitted work. 


\begin{abstract}
Androgenetic alopecia (AGA) is characterized by non-scarring follicle miniaturization. Despite the success of approved therapies, commonly reported side effects and the need for continual use has led to the investigation of alternative therapies. The aim of this paper is to critically review the success of off-label, topical monotherapies for treatment of AGA in men. A literature search was conducted to obtain randomized, controlled and blinded studies that investigated off-label, topical, monotherapies in male patients. Hair density, hair diameter and hair growth were used to evaluate treatment success. Fourteen off-label topical therapies were investigated among the 16 studies that met inclusion criteria. Nine off-label therapies were reported to produce a significantly greater improvement in hair restoration parameters (e.g., mean change from hair count and hair diameter) as compared to placebo ( $p<0.05$ for all treatments). In two studies, procyanidin oligomers exhibited greater efficacy over vehicle with response to mean change in hair density (hairs $\left./ \mathrm{cm}^{2}\right)\left(p_{s}<0.0001\right.$ at week 24$)$. In conclusion, prostaglandin analogs and polyphenols, such as latanoprost and procyanidin oligomers, can improve hair restoration parameters in male AGA patients, possibly through targeting mechanisms proposed in the etiology of AGA. The current evidence suggests short term (24 weeks) use may provide benefit for hair loss patients; however, long-term efficacy and safety data are required.
\end{abstract}

\title{
Key Points
}

- Due to the potential side effects and the need for continual use of approved therapies, there is a need to explore the efficacy of off-label options.

- Eight off-label topical therapies, including prostaglandin analogs and polyphenols, were found to improve hair density, while one additional therapy improved hair diameter.

Keywords: androgenetic alopecia, off-label therapies, prostaglandin analogs, polyphenols 


\subsection{Introduction}

Androgenetic alopecia (AGA) is a common hair loss disorder characterized by non-scarring follicle miniaturization ${ }^{1}$. The etiology of AGA is multifactorial and polygenetic, with androgens playing a pivotal role $\mathrm{e}^{2,3}$. Finasteride, an oral $5-\alpha$ reductase inhibitor, and minoxidil, a topical vasodilator, are FDA and Health Canada approved for treatment of $A G A^{4-7}$. Despite their success, these approved therapies have several drawbacks. Their improvement in hair restoration parameters tends to plateau after a few years of continued use. In addition, these approved therapies have been associated with side effects such as skin irritation and sexual dysfunction ${ }^{4-7}$. Thus, there is a need for new, safe and effective treatments for AGA. The aim of this paper is to critically review the success of off-label, topical therapies for treatment of androgenetic alopecia in men.

\subsection{Methods of Literature Search}

A literature search in PubMed, MEDLINE (Ovid), Embase (Ovid), and CINAHL was conducted using the following search terms: 'androgenetic alopecia', 'male pattern baldness', 'AGA' and 'randomized controlled trial' (February 2018). Randomized, controlled (placebo or vehicle) and blinded (single, double or triple) trials that investigated topical off-label monotherapies for the treatment of androgenetic alopecia in male patients were included. Studies that investigated oral or combination therapies, included only female patients, and/or included alopecia conditions other than androgenetic alopecia were excluded. Eligible studies were required to evaluate treatment success using hair density $\left(\right.$ hair $\left./ \mathrm{cm}^{2}\right)$, hair diameter $(\mathrm{mm})$ or hair growth $\left(\mathrm{mm} / \mathrm{cm}^{2}\right)$. Clinical outcomes vary widely among studies; we selected a range of quantitative measurements to be as inclusive and objective as possible.

\subsection{Results of Literature Search}

Sixteen studies met inclusion criteria (Figure 1$)^{8-23}$. A total of 1,709 male participants were included with an average age of 40 years. Fourteen off-label topical therapies were investigated: a 
penta-peptide (Gly-Pro-lie-Gly-Ser), adenosine, bimatoprost, Curcuma aeruginosa extract, dabao, fulvestrant, a herbal extract, latanoprost, a non-steroidal anabolic hormone gel, procyanidin oligomers, pyrithione zinc, roxithromycin, sodium valproate and viprostol (Tables $1-3)^{8-23}$. Treatment duration ranged from 4 weeks $^{23}$ to 52 weeks ${ }^{21}$ with 24 weeks the most common duration $(10 / 16=63 \%)^{9,12,15-19,21-}$ ${ }^{23}$. Investigated therapies were administered in a wide array of formulations including cream (herbal extract ${ }^{11}$ ), gel (non-steroidal anabolic hormones ${ }^{18}$ ), lotion (adenosine, penta-peptide, dabao, roxithromycin ${ }^{12-14,17}$ ), shampoo $\left(\right.$ pyrithione zinc $^{8}$ ), solution (bimatoprost ${ }^{22-23}$, latanoprost ${ }^{9}$, curcuma aeruginosa extract ${ }^{20}$, fulvestrant ${ }^{10}$, procyanidin $^{16,21}$, viprostal $^{19}$ ), and spray (sodium valproate ${ }^{15}$ ).

\subsection{Hair Density}

Fifteen included studies used hair density to evaluate treatment success of off-label therapies in AGA patients ${ }^{8-11,13,15-23}$. The mean change in hair density (hairs $/ \mathrm{cm}^{2}$ ) from baseline to end of treatment for off-label therapies ranged from - 4 hairs $/ \mathrm{cm}^{2}$ to 54 hairs $/ \mathrm{cm}^{2}$ (Table 1). Eight off-label therapies (pyrithione zinc shampoo, latanoprost, a herbal extract, adenosine, sodium valproate spray, procyanidin oligomers, dabao, and a non-steroidal anabolic hormone solution) had a significantly higher mean change from baseline as compared to placebo ( $p<0.05$ for all treatments $)^{8,9,11,13,15-18,21}$. The highest mean change in hair density (hairs $/ \mathrm{cm}^{2}$ ) after 24 weeks of treatment was found with latanoprost (Figure 2). Two studies reported procyanidin oligomer treatment exhibited greater efficacy over vehicle with response to mean change in hair density $\left(\right.$ hairs $\left./ \mathrm{cm}^{2}\right)$ by end of 24 weeks of treatment $\left(p_{s}<0.001\right)^{16,21}$.

\subsection{Hair Diameter}

Two included studies used changes in hair diameter to evaluate treatment success of off-label therapies in AGA patients (Table 2) ${ }^{8,12}$. Mean change in hair diameter $(\mathrm{mm}$ ) as compared to baseline was significantly higher with roxithromycin as compared to vehicle after 24 weeks of treatment $(p<0.01)^{12}$, whereas, 26 weeks of pyrithione zinc treatment was not significantly different from placebo treatment ${ }^{8}$. 


\subsection{Hair Growth}

Three studies measured the impact of off-label topical therapy on mean change in hair growth, as defined as $\mathrm{mm} / \mathrm{cm}^{2}$ from baseline, compared to vehicle treated patients (Table 3$)^{10,22,23}$. Bimatoprost treatment once or twice a day produced a mean change in hair growth after 24 weeks of treatment of $0.76 \mathrm{~mm} / \mathrm{cm}^{2}$ and $0.92 \mathrm{~mm} / \mathrm{cm}^{2}$, respectively ${ }^{22,23}$. A significantly lower mean change in hair growth was found with fulvestrant as compared to minoxidil after 16 weeks of treatment $(p<0.001)^{10}$.

\subsection{Discussion}

In recent years, many novel treatments for treating AGA and other hair loss have been investigated. The objective of this review was to evaluate therapeutic, off-label options available to treat AGA. The existing pharmaceutical therapies for AGA are oral finasteride and topical minoxidil; some of the off-label treatments discussed in this review work via similar mechanisms. Eight off-label therapies (pyrithione zinc shampoo, latanoprost, a herbal extract, adenosine, sodium valproate spray, procyanidin oligomers, dabao, a non-steroidal anabolic hormone solution) produced significant increases in hair density, while roxithromycin significantly increased hair diameter ${ }^{8,9,11-13,15-18,21}$. Apart from placebo/vehicle, minoxidil $5 \%$ was used as an active comparator in three studies, where it was significantly better than fulvestrant ${ }^{10}$, numerically similar to pyrithione zinc shampoo ${ }^{8}$ and $C$. aeruginosa extract $^{20}$, and numerically better than bimatoprost formulations ${ }^{22}$. Although safety was not a focus of this review, all but four studies ${ }^{8,17-19}$ monitored adverse events. There were no adverse events or adverse events related to treatment for herbal extract, adenosine, procyanidin oligomers, and roxithromycin. Studies of bimatoprost, latanoprost, fulvestrant, herbal extract, valproate, and Curcuma aeruginosa reported mild skin-related adverse events such as erythema, pruritus, urticarial, or dermatitis. Most of the treatments appear to be well-tolerated and safe in the short-term. 
Of the 24 week treatments, latanoprost, a prostaglandin analog antagonist, was found to have the highest mean change in hair density (hairs $\left./ \mathrm{cm}^{2}\right)^{9}$. Prostaglandin analogs, which include latanoprost, bimatoprost and viprostol, can stimulate keratinocyte and melanocyte activity, enhancing hair growth and pigmentation ${ }^{24}$. Interestingly, viprostol, a synthetic prostaglandin inhibitor, was not an effective treatment ${ }^{19}$. This finding could be due to the synthetic nature of viprostol, the hair loss severity of included patients (IIlv to V) or the dosage used.

In two studies, procyanidin oligomers exhibited a greater efficacy over vehicle by end of treatment with response to mean change in hair density (hairs $/ \mathrm{cm} 2)^{16,21}$. Procyanidin oligomers, and other polyphenols, most likely promote hair growth via nitric oxide, a gas produced in the lining of veins which promotes vasodilation and reduces cholesterol build-up. Polyphenols have also been shown to inhibit $5 \alpha$-reductase activity which has been theorized to play a role in the pathogenesis of AGA ${ }^{25}$.

Regardless of these promising effects, more clinical trials need to be conducted before the use of these off-label treatments is sanctioned. Of particular note is that the current evidence for off-label treatments is from clinical studies that are short in duration, with 24 weeks the most common. Longterm efficacy and safety data are not available and we are thus unable to draw comparisons with approved therapies. While improvement can plateau over time with finasteride and minoxidil, the longterm efficacy of off-label treatments is unknown. The patients in the included studies were predominantly male and differences in treatment efficacy between males and females should be kept in mind. Therapies with multi-factorial capabilities may be more appropriate for treating female pattern hair loss, whereas, male pattern hair loss patients benefit more from a direct anagen effect ${ }^{2}$.

Additionally, an important limitation when evaluating efficacy of hair loss treatments is the variety of outcome measures used to measure successful hair growth; there is no accepted industry standard. Assessment of global photographs by an expert, hair counts using phototrichogram, and 
manual hair counts using clippings or hair density in a target area were all reported in studies. Global assessments based on photographs, for example, can offer useful insight, but they are not the same as blinded quantitative measurement. Hair count can be converted into hair density when a target area is provided by the authors, but not all authors disclosed this information. This should be a point of consideration in the development of new trials as a consistent quantitative outcome measure can aid in comparing different treatments. Patient-centred outcomes such as satisfaction are also important. While the reported data (Tables 1-3) certainly show an increase in hair parameters, increases may not be noticeable or satisfactory for patients to justify treatment or, patients may express satisfaction despite little to no clinical improvement. Even if patients believe a treatment is beneficial, a case can be made that clinicians should not encourage use of treatments that do not objectively improve hair loss.

\subsection{Conclusion}

Preliminary evidence suggests that, in the short-term, eight off-label therapies such as

prostaglandin analogs and polyphenols can significantly improve hair density ${ }^{8,9,11,13,15-18,21}$. Further study of hair loss patients enrolled in randomized, controlled and blinded studies is needed to expand on the efficacy and safety data that has been collected to date. In the meantime, clinicians may wish to use off-label therapies as they may provide benefit to patients. Hair loss needs should be addressed individually as both off-label and approved treatment that works for one person may not work for another. 


\subsection{References}

1. Crabtree JS, Kilbourne EJ, Peano BJ, Chippari S, Kenney T, McNally C, et al. A mouse model of androgenetic alopecia. Endocrinology. 2010;151(5):2373-80.

2. Sadick NS, Callender VD, Kircik LH, Kogan S. New Insight Into the Pathophysiology of Hair Loss Trigger a Paradigm Shift in the Treatment Approach. Journal of Drugs in Dermatology. 2017;16(11):13540.

3. Christoph T, Müller-Röver S, Audring H, Tobin DJ, Hermes B, Cotsarelis G, et al. The human hair follicle immune system: cellular composition and immune privilege. British Journal of Dermatology. 2000;142(5):862-73.

4. ROGAINE ${ }^{\circledR}$ MINOXIDIL TOPICAL 2\% SOLUTION [Internet]. Government of Canada. 2017 [cited 2018 Sep 18]. Available from: https://pdf.hres.ca/dpd_pm/00040937.PDF

5. Men's Rogaine [Internet]. Drugs@FDA: FDA Approved Drug Products. 2006 [cited 2018 Sept 18]. Available from: https://www.accessdata.fda.gov/drugsatfda_docs/label/2006/021812s000LBL.pdf

6. PROPECIA ${ }^{\circledast}$ (finasteride) tablets for oral use [Internet]. FDA U.S. Food and Drug Administration. 2016 [cited 2018 Sep 18]. Available from:

https://www.accessdata.fda.gov/drugsatfda_docs/label/2014/020788s024lbl.pdf

7. PROPECIA ${ }^{\circledR}$ Finasteride tablets, USP Film-coated Tablets 1 mg Type II $5 \alpha$-reductase inhibitor [Internet]. Government of Canada. 2018 [cited 2018 Sep 18]. Available from: https://pdf.hres.ca/dpd_pm/00043972.PDF

8. Berger RS, Fu JL, Smiles KA, Turner CB, Schnell BM, Werchowski KM, et al. The effects of minoxidil, $1 \%$ pyrithione zinc and a combination of both on hair density: a randomized controlled trial. British Journal of Dermatology. 2003;149(2):354-62.

9. Blume-Peytavi U, Lönnfors S, Hillmann K, Garcia Bartels N. A randomized double-blind placebocontrolled pilot study to assess the efficacy of a 24 -week topical treatment by latanoprost $0.1 \%$ on hair growth and pigmentation in healthy volunteers with androgenetic alopecia. Journal of the American Academy of Dermatology. 2012;66(5):794-800.

10. Gassmueller J, Hoffmann R, Webster A. Topical fulvestrant solution has no effect on male and postmenopausal female androgenetic alopecia: results from two randomized, proof-of-concept studies. British Journal of Dermatology. 2008;158(1):109-15.

11. Greenberg JH, Katz M. Treatment of androgenetic alopecia with a $7.5 \%$ herbal preparation: Journal of Dermatological Treatment. 1996;7(3):159-62. 
12. Ito T, Fukamizu H, Ito N, Seo N, Yagi H, Takigawa M, et al. Roxithromycin antagonizes catagen induction in murine and human hair follicles: implication of topical roxithromycin as hair restoration reagent. Archives of Dermatological Research. 2009;301(5):347-55.

13. Iwabuchi T, Ideta R, Ehama R, Yamanishi H, lino M, Nakazawa Y, et al. Topical adenosine increases the proportion of thick hair in Caucasian men with androgenetic alopecia. Journal of Dermatology. 2016;43(5):567-70.

14. Iwabuchi T, Takeda S, Yamanishi H, Ideta R, Ehama R, Tsuruda A, et al. The topical penta-peptide Gly-Pro-Ile-Gly-Ser increases the proportion of thick hair in Japanese men with androgenetic alopecia. Journal of Cosmetic Dermatology. 2016;15(2):176-84.

15. Jo SJ, Shin H, Park YW, Paik SH, Park WS, Jeong YS, et al. Topical valproic acid increases the hair count in male patients with androgenetic alopecia: a randomized, comparative, clinical feasibility study using phototrichogram analysis. Journal of Dermatology. 2014;41(4):285-91.

16. Kamimura A, Takahashi T, Watanabe Y. Investigation of topical application of procyanidin B-2 from apple to identify its potential use as a hair growing agent. Phytomedicine International Journal of Phytotherapy and Phytopharmacology. 2000;7(6):529-36.

17. Kessels AG, Cardynaals RL, Borger RL, Go MJ, Lambers JC, Knottnerus JA, et al. The effectiveness of the hair-restorer 'Dabao' in males with alopecia androgenetica. A clinical experiment. Journal of Clinical Epidemiology. 1991;44(4-5):439-47.

18. Lindenbaum ES, Feitelberg AL, Tendler M, Beach D, Gamliel-Lazarovich A, Har-Shai Y, et al. Pilot study of a novel treatment for androgenetic alopecia using enriched cell culture medium: clinical trials. Dermatology Online Journal. 2003;9(1):4.

19. Olsen EA, DeLong E. Transdermal viprostol in the treatment of male pattern baldness. Journal of the American Academy of Dermatology. 1990;23(3):470-2.

20. Pumthong G, Asawanonda $P$, Varothai $S$, Jariyasethavong $V$, Triwongwaranat $D$, Suthipinittharm $P$, et al. Curcuma aeruginosa, a novel botanically derived $5 \alpha$-reductase inhibitor in the treatment of male-pattern baldness: a multicenter, randomized, double-blind, placebo-controlled study. Journal of Dermatological Treatment. 2012;23(5):385-92.

21. Takahashi T, Kamimura A, Kagoura M, Toyoda M, Morohashi M. Investigation of the topical application of procyanidin oligomers from apples to identify their potential use as a hair-growing agent. Journal of Cosmetic Dermatology. 2005;4(4):245-9.

22. Safety and Efficacy Study of Bimatoprost in the Treatment of Men With Androgenic Alopecia Study Results - ClinicalTrials.gov [Internet]. [cited 2018 Sept 18]. Available from: https://clinicaltrials.gov/ct2/show/results/NCT01325337 
23. A Safety and Efficacy Study of Bimatoprost in Men With Androgenic Alopecia (AGA) - Study Results - ClinicalTrials.gov [Internet]. [cited 2018 Sept 18]. Available from:

https://clinicaltrials.gov/ct2/show/results/NCT01904721

24. Johnstone M, Albert D. Prostaglandin-Induced Hair Growth. Survey of Ophthalmology. 2002;47(1):S185-202.

25. Hiipakka RA, Zhang H-Z, Dai W, Dai Q, Liao S. Structure-activity relationships for inhibition of human 5alpha-reductases by polyphenols. Biochemical Pharmacology. 2002;63(6):1165-76. 


\section{Figure Captions:}

Figure 1: Study Selection Process

Figure 2: Mean Change in Hair Density (hairs $/ \mathrm{cm}^{2}$ ) after 24 weeks of Off-Label Topical Therapy, ${ }^{8,9,13-22}$. Bimatoprost A, B, and C: Details on these treatment groups (e.g., percentage of bimatoprost) not yet released on clinicaltrials.gov; Dabao: $50 \%$ ethanol, $42 \%$ water, $8 \%$ Chinese herbal extracts (saffron flowers, mulberry leaves, stemona root, fruits of the pepper plant, sesame leaves, the skin of the fruit of Sichuan pepper, ginger root, Chinese angelica root, bark of the Pseudolarix, fruits of the hawthorn); Latanoprost: $50 \%$ ethanol, $20 \%$ propylene glycol water; Non-steroidal anabolic hormones (NSAH): CMM, made of insulin, thyroxin and growth hormone gelled in $1.5 \%$ hydroxyethyl cellulose; Procyanidin B-2: $70 \%$ ethanol, 10\% 1,3-butylene glycol, $0.5 \% \mathrm{~N}$-acetylglutamine-isostearyl ester, $0.25 \%$ polyoxyethylene glyceryl monopyroglutamate monoisosterate, $0.1 \% \mathrm{dl}$ - $\alpha$-tocopherol, $0.05 \% \mathrm{~d}$ biotin, $0.1 \%$ ascorbyl palmitate, $0.001 \% \beta$-carotene, $0.1 \%$ sodium citrate and $17.899 \%$ purified water; Procyanidin Mix: 7.3\% procyanidin B-1, 26.2\% procyanidin B-2, 7.7\% procyanidin C-1, $40 \%$ other oligomeric procyanidins, $70 \%$ ethanol, 3\% 1,3-butylene glycol, $0.15 \% \mathrm{~N}$-acetylglutamine isostearyl ester, $0.067 \%$ citrate-sodium citrate buffer, $0.05 \%$ sodium bisulfite, purified water; Sodium Valproate: $8.3 \%$ sodium valproate using a $27 \%$ ethanol solution as the vehicle. 
Table 1: Impact of Off-Label Topical Therapy on Hair Density (hairs $/ \mathrm{cm}^{2}$ ) by End of Treatment

Study

Characteristics

$\mathrm{N}: 200$

Berger et al. $2003^{8}$

Blume-Peytavi et al. $2012^{9}$

Gassmueller et al. $2008^{10}$

Greenberg \& Katz $1996^{11}$

Iwabuchi et al. $2016 \mathrm{a}^{13}$

Jo et al. $2014^{15}$

Kamimura et al. $2000^{16}$

Kessels et al. $1991^{17}$

Lindenbaum et al. $2003^{18}$

NCT01325337 22

NCT01904721 23

Olsen \& DeLong et al. $1990^{19}$

Pumthong et al. $2012^{20}$

Takahashi et al. $2005^{21}$

Age: 40

$$
\mathrm{N}: 16
$$

Age: (23-35)

$\mathrm{N}: 102$

Age: 38

$$
\begin{gathered}
\mathrm{N}: 24 \\
\text { Age: } 43
\end{gathered}
$$

$\mathrm{N}: 38$

Age: 42

$\mathrm{N}: 40$

Age: 38

$\mathrm{N}: 30$

Age: 47 $\mathrm{N}: 396$

N: 56

Age: 28

N: 307

\section{$\mathrm{N}: 244$}

$\mathrm{N}: 72$

Age: 38

N: 89

Age: 39

Severity: II-VII

$$
\mathrm{N}: 49
$$

Age: (27-58)
Treatment Details

Placebo Shampoo (1x day)

Severity: IIIv to IV Duration: 26 wks

Severity: II to III

Duration: 24 wks

Severity: III to Va

Duration: 16 wks

Severity: III to IV

Duration: 40 wks

Duration: 24 wks

Severity: IIIv to V

Duration: 24 wks

Duration: 24 wks

Duration: 24 wks

Severity: II to IV

Duration: 24 wks

Duration: 24 wks

Duration: 7-24 wks

Severity: Illv to V

Duration: 24 wks

Duration: 24 wks

Duration: $24-52$ wks

Adenosine $(0.75 \%)$

Placebo (1\%, $1.8 \mathrm{~mL} 2 \mathrm{x}$ day)

Placebo (1.7 mL, $2 x$ day)

Dabao (1.7 mL, 2x day)

Placebo (1-3 mL/day)

Vehicle (1x day) Minoxidil (5\%, 1x day)

Vehicle ( $2 x$ day)

Vehicle (2x day)

Minoxidil (5\%, 2x day)

day)

Vehicle (2 $\mathrm{mL} 2 \mathrm{x}$ day)

\begin{tabular}{|c|c|} 
Treatment Details & \\
\hline Pyrithione Zinc Shampoo (1\%, 1x day) & \\
\hline Pyrithione Zinc Shampoo (1\%, 1x day) + Minoxidil (5\%, 2x day) & \\
\hline Minoxidil (5\%, 2x day) + Placebo Shampoo (1x day) & \\
\hline Placebo $(50 \mu \mathrm{L} /$ day) & \\
\hline Latanoprost $(0.1 \%, 50 \mu \mathrm{L} /$ day) & \\
\hline Vehicle $(2 x$ day) & $\mathrm{mg} /$ day) \\
\hline Fulvestrant $(70 \mathrm{mg}, 42 \mathrm{mg}$ ) & \\
\hline Minoxidil $(20 \mathrm{mg} / \mathrm{mL}, 12 \mathrm{mg} /$ day) &
\end{tabular}

Mean Change From Baseline

-1 hairs $/ \mathrm{cm}^{2}$

6 hairs $/ \mathrm{cm}^{2} *$

6 hairs $/ \mathrm{cm}^{2 *}$

12 hairs $/ \mathrm{cm}^{2} *$

8 hairs $/ \mathrm{cm}^{2}$

51 hairs $/ \mathrm{cm}^{2} *$

8 hairs $/ \mathrm{cm}^{2}$

8 hairs $/ \mathrm{cm}^{2}$

25 hairs $/ \mathrm{cm}^{2} * *$

6 hairs $/ \mathrm{cm}^{2}$

54 hairs $/ \mathrm{cm}^{2} *$

-10 hairs $/ \mathrm{cm}^{2}$

12 hairs $/ \mathrm{cm}^{2} *$

Vehicle $(0.8 \mathrm{~mL} /$ dose, $2 \mathrm{x}$ day)

Sodium Valproate Spray ( $0.8 \mathrm{~mL} /$ dose, $2 x$ day)

Procyanidin B-2 (1\%, $1.8 \mathrm{~mL} 2 x$ day)

Non-Steroidal Anabolic Hormones (1-3 mL/day)

Bimatoprost Formulation $B$ (1 $x$ day)

Bimatoprost Formulation C (1x day)

Bimatoprost Formulation A (1x day)

Bimatoprost Solution 1 ( $2 x$ day)

Bimatoprost Solution 2 ( $2 x$ day)

Vehicle $(0.3 \mathrm{~mL} /$ dose, $2 x$ day)

Viprostol ( $120 \mu \mathrm{g}, 0.3 \mathrm{~mL} /$ dose, $2 \mathrm{x}$ day)

Placebo ( $0.3 \mathrm{~mL} /$ dose, $2 x$ day)

Curcuma aeruginosa extract (5\%, $2 x$ day)

Minoxidil $(5 \%, 2 x$ day) + Curcuma aeruginosa extract $(5 \%, 2 x$

Procyanidin (0.7\%, $2 \mathrm{~mL} 2 \mathrm{x}$ day)
-1 hairs $/ \mathrm{cm}^{2 a}$

23 hairs $/ \mathrm{cm}^{2 \mathrm{a} *}$

4 hairs $/ \mathrm{cm}^{2}$

28 hairs $/ \mathrm{cm}^{2} *$

22 hairs $/ \mathrm{cm}^{2}$

27 hairs $/ \mathrm{cm}^{2} *$

30 hairs $/ \mathrm{cm}^{2} *$

4 hairs $/ \mathrm{cm}^{2 b}$

6 hairs $/ \mathrm{cm}^{2 \mathrm{~b}}$

6 hairs $/ \mathrm{cm}^{2 \mathrm{~b}}$

13 hairs $/ \mathrm{cm}^{2 b}$

13 hairs $/ \mathrm{cm}^{2 b}$

9 hairs $/ \mathrm{cm}^{2 b}$

6 hairs $/ \mathrm{cm}^{2 \mathrm{~b}}$

-8 hairs $/ \mathrm{cm}^{2}$

-4 hairs $/ \mathrm{cm}^{2}$

-3 hairs $/ \mathrm{cm}^{2}$

20 hairs $/ \mathrm{cm}^{2}$

31 hairs $/ \mathrm{cm}^{2}$

31 hairs $/ \mathrm{cm}^{2}$

33 hairs $/ \mathrm{cm}^{2}$

-8 hairs $/ \mathrm{cm}^{2}$

6 hairs $/ \mathrm{cm}^{2} *$
16 hairs $/ \mathrm{cm}^{2}$

22 hairs $/ \mathrm{cm}^{2 b}$

${ }^{*} \mathrm{p}<0.05$ as compared to placebo / vehicle; ${ }^{* *} \mathrm{p}<0.05$ as compared to comparator; a: median change; $\mathbf{b}$ : terminal hairs were used; Age (years): Mean (range); Severity:

Based on Hamilton-Norwood Scale and eligibility criteria; Wks: weeks; Latanoprost: $50 \%$ ethanol, $20 \%$ propylene glycol water; Herbal extract: $7.5 \%$ extract of fennel, 
polygonum, mentha, chamomile, thuja and hibiscus in a water-based cream; Sodium Valproate Spray: $8.3 \%$ sodium valproate using a $27 \%$ ethanol solution as the vehicle; Procyanidin B-2: 70\% ethanol, 10\% 1,3-butylene glycol, 0.5\% N-acetylglutamine-isostearyl ester, 0.25\%polyoxyethylene glyceryl monopyroglutamate monoisosterate,

$0.1 \% \mathrm{dl}$ - $\alpha$-tocopherol, $0.05 \% \mathrm{~d}$-biotin, $0.1 \%$ ascorbyl palmitate, $0.001 \% \beta$-carotene, $0.1 \%$ sodium citrate and $17.899 \%$ purified water; Procyanidin: $7.3 \%$ procyanidin B-1,

$26.2 \%$ procyanidin $\mathrm{B}-2,7.7 \%$ procyanidin $\mathrm{C}-1,40 \%$ other oligomeric procyanidins, $70 \%$ ethanol, $3 \% 1,3$-butylene glycol, $0.15 \% \mathrm{~N}$-acetylglutamine isostearyl ester, $0.067 \%$

citrate-sodium citrate buffer, $0.05 \%$ sodium bisulfite, purified water; Dabao: $50 \%$ ethanol, $42 \%$ water, $8 \%$ Chinese herbal extracts (saffron flowers, mulberry leaves,

stemona root, fruits of the pepper plant, sesame leaves, the skin of the fruit of Sichuan pepper, ginger root, Chinese angelica root, bark of the Pseudolarix, fruits of the

hawthorn); Non-steroidal anabolic hormones: CMM, made of insulin, thyroxin and growth hormone gelled in 1.5\% hydroxyethyl cellulose; Bimatoprost A, B, C, 1 and 2:

Details on these treatment groups (e.g., percentage of bimatoprost) not yet released on clinicaltrials.gov 
Table 2: Impact of Off-Label Topical Therapy on Hair Diameter $(\mathrm{mm})$ by End of Treatment ${ }^{8,14}$

\begin{tabular}{|c|c|c|c|}
\hline Study & Characteristics & Treatment Details & Mean Change from Baseline \\
\hline \multirow{4}{*}{ Berger et al. $2003^{8}$} & \multirow{4}{*}{$\begin{array}{c}\mathrm{N}: 200 \\
\text { Age: } 40 \\
\text { Severity: IIlv to IV } \\
\text { Duration: } 26 \mathrm{wks}\end{array}$} & Placebo Shampoo (1x day) & $-0.04 \mathrm{~mm}$ \\
\hline & & Pyrithione Zinc Shampoo (1\%, $1 x$ day) & $-0.04 \mathrm{~mm}$ \\
\hline & & Pyrithione Zinc Shampoo ( $1 \%, 1 x$ day) + Minoxidil (5\%, $2 x$ day) & $-0.01 \mathrm{~mm}$ \\
\hline & & Minoxidil (5\%, $2 x$ day) + Placebo Shampoo ( $1 x$ day) & $-0.01 \mathrm{~mm}$ \\
\hline \multirow[b]{2}{*}{ Ito et al. $2009^{12}$} & \multirow{2}{*}{$\begin{array}{c}\text { N: } 24 \\
\text { Age: } 48 \\
\text { Severity: II to IIIV } \\
\text { Duration: } 24 \mathrm{wks}\end{array}$} & Vehicle & $0.0004 \mathrm{~mm}$ \\
\hline & & Roxithromycin & $0.309 \mathrm{~mm} *$ \\
\hline
\end{tabular}


Table 3: Impact of Off-Label Topical Therapy on Hair Growth $\left(\mathrm{mm} / \mathrm{cm}^{2}\right)$ by End of Treatment ${ }^{10,24,25}$

\begin{tabular}{|c|c|c|c|}
\hline Study & Characteristics & Treatment Details & Mean Change from Baseline \\
\hline \multirow{3}{*}{ Gassmueller et al. $2008^{10}$} & \multirow{3}{*}{$\begin{array}{c}\text { N: } 102 \\
\text { Age: } 38 \\
\text { Severity: III to Va } \\
\text { Duration: } 16 \text { wks }\end{array}$} & Vehicle ( $2 x$ day) & $0.7 \mathrm{~mm} / \mathrm{cm}^{2}$ \\
\hline & & Fulvestrant (70 mg/mL, $42 \mathrm{mg} /$ day) & $0.84 \mathrm{~mm} / \mathrm{cm}^{2}$ \\
\hline & & Minoxidil ( $20 \mathrm{mg} / \mathrm{mL}, 12 \mathrm{mg} /$ day) & $2.9 \mathrm{~mm} / \mathrm{cm}^{2} * \wedge$ \\
\hline \multirow{5}{*}{ NCT01325337 22} & \multirow{5}{*}{$\begin{array}{l}\text { N: } 307 \\
\text { Duration: } 24 \text { wks }\end{array}$} & Bimatoprost Formulation C (1x day) & $0.12 \mathrm{~mm} / \mathrm{cm}^{2}$ \\
\hline & & Vehicle (1x day) & $0.13 \mathrm{~mm} / \mathrm{cm}^{2}$ \\
\hline & & Bimatoprost Formulation B (1x day) & $0.25 \mathrm{~mm} / \mathrm{cm}^{2}$ \\
\hline & & Bimatoprost Formulation A (1x day) & $0.76 \mathrm{~mm} / \mathrm{cm}^{2}$ \\
\hline & & Minoxidil $(5 \%, 1 \times$ day) & $1.29 \mathrm{~mm} / \mathrm{cm}^{2}$ \\
\hline \multirow{3}{*}{ NCT01904721 23} & \multirow{3}{*}{$\begin{array}{c}\mathrm{N}: 244 \\
\text { Duration: } 7-24 \text { wks }\end{array}$} & Vehicle ( $2 x$ day) & $0.05 \mathrm{~mm} / \mathrm{cm}^{2}$ \\
\hline & & Bimatoprost Solution 2 ( $2 x$ day) & $0.67 \mathrm{~mm} / \mathrm{cm}^{2}$ \\
\hline & & Bimatoprost Solution 1 ( $2 x$ day) & $0.92 \mathrm{~mm} / \mathrm{cm}^{2}$ \\
\hline $\begin{array}{l}{ }^{*} \mathrm{p}<0.05 \text { as compared to plac } \\
\text { and eligibility criteria; Wks: } \\
\text { clinicaltrials.gov }\end{array}$ & & off-label topical the & $\begin{array}{l}\text { Jamilton-Norwood Scale } \\
\text { prost) not yet released on }\end{array}$ \\
\hline
\end{tabular}


Figure 1.

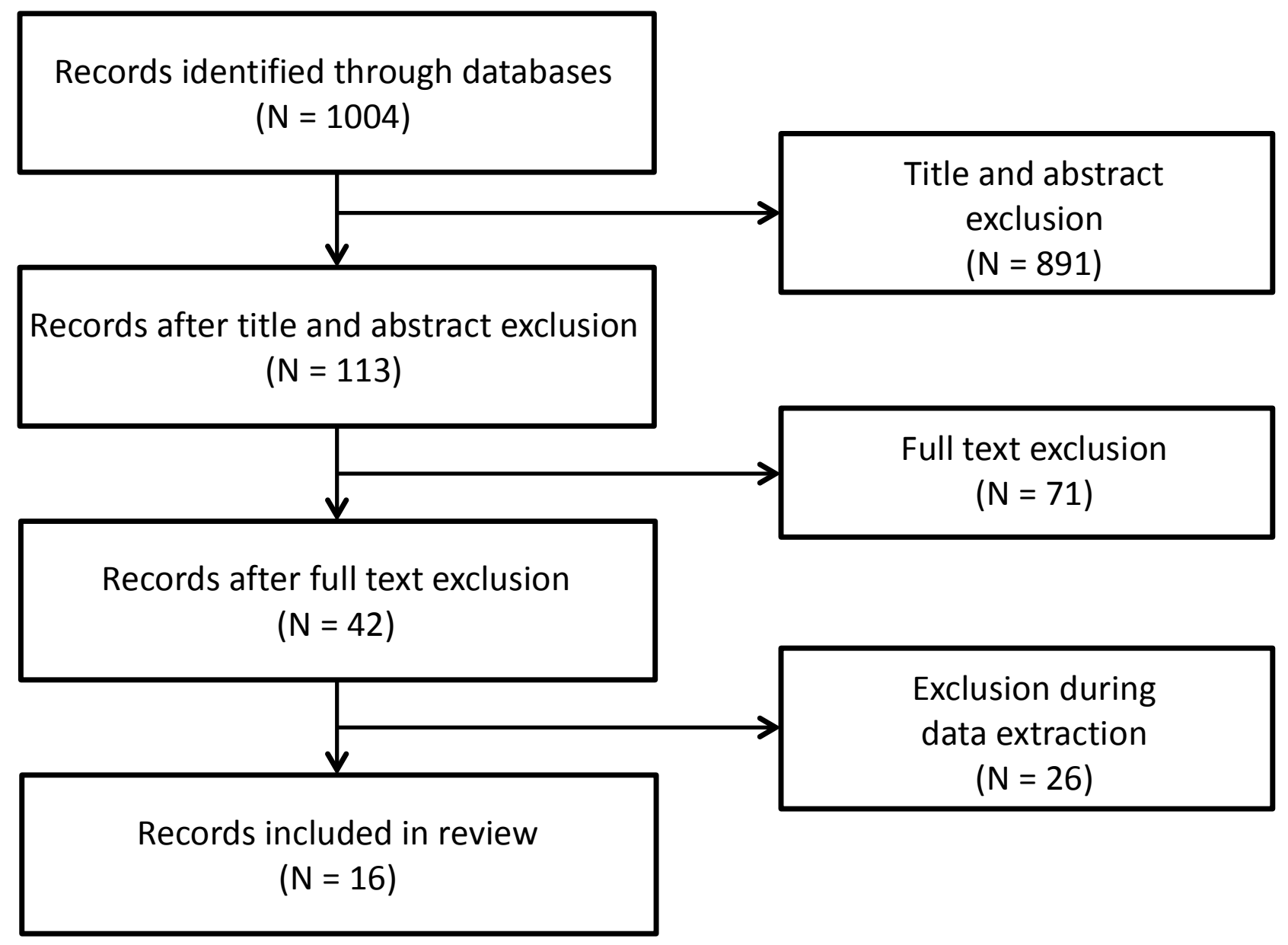


Figure 2

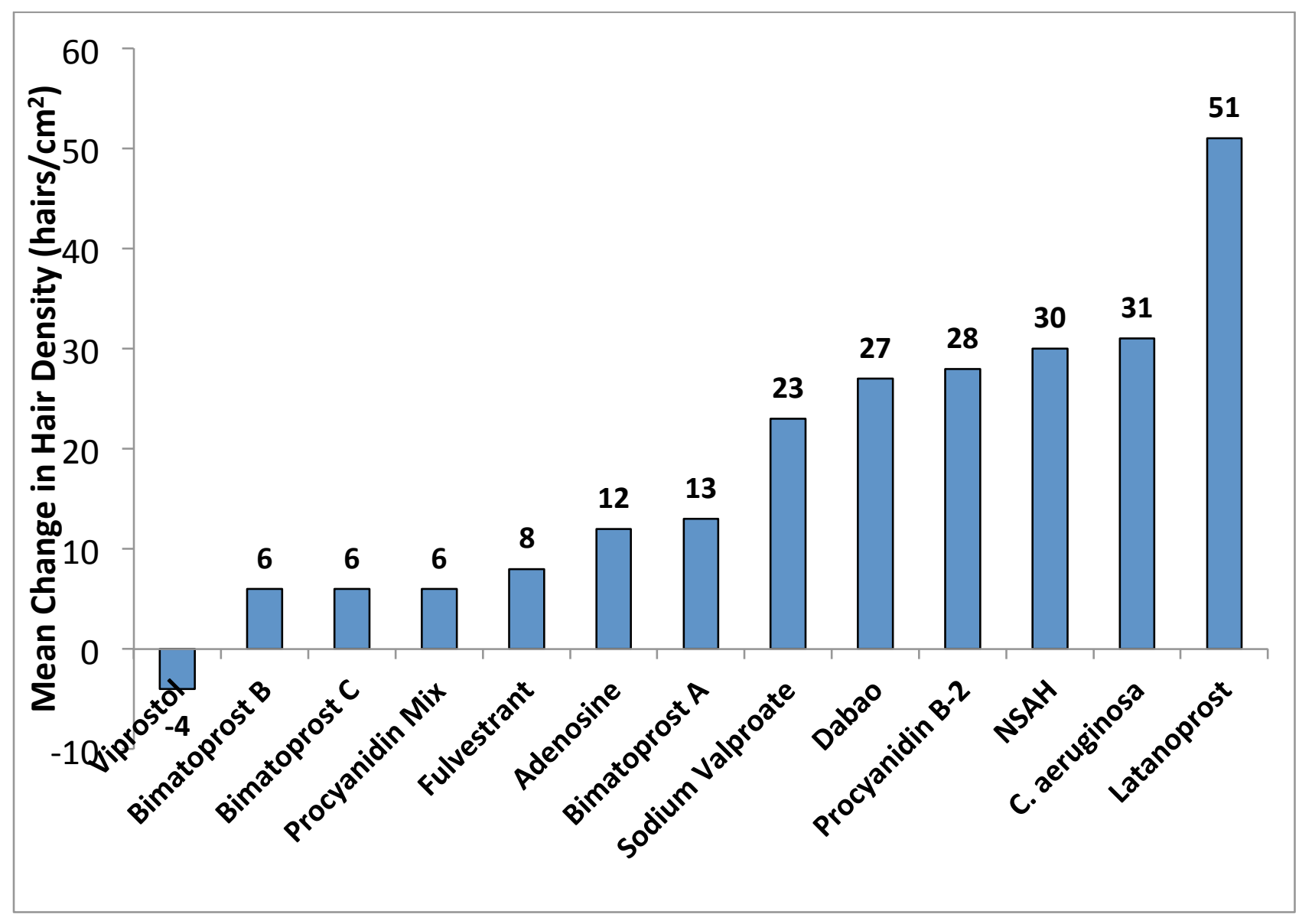

